\title{
The Readiness Potential reflects planning-based expectation, not uncertainty, in the timing of action
}

\author{
Eoin Travers ${ }^{1}$ \\ Maja Friedemann ${ }^{1,2}$ \\ Patrick Haggard ${ }^{1}$ \\ ${ }^{1}$ Institute of Cognitive Neuroscience, University College London \\ ${ }^{2}$ Current address: Department of Experimental Psychology, University of Oxford
}

\begin{abstract}
Actions are guided by a combination of external cues, internal intentions and stored knowledge. Self-initiated voluntary actions, produced without any immediate external cue, may be preceded by a slow EEG Readiness Potential (RP) that progressively increases prior to action. The cognitive significance of this neural event is controversial. Some accounts link the RP to the fact that timing of voluntary actions is generated endogenously, without external constraints, and perhaps even randomly. Other accounts take the RP as reflecting the unique role of a planning process, and therefore of temporal expectation, in voluntary actions. In many previous experiments, actions are both unconstrained by external cues, but also potentially involve preplanning and anticipation. To separate these factors, we developed a reinforcement learning paradigm where participants learned, through trial and error, the optimal time to act. If the RP reflects freedom from external constraint, its amplitude should be greater early in learning, when participants do not yet know the best time to act. Conversely, if the RP reflects planning, it should be greater later on, when participants have learned, and know in advance, the time of action. We found that RP amplitudes grew with learning, suggesting that this neural activity reflects planning and anticipation for the forthcoming action, rather than freedom from external constraint.
\end{abstract}

Word Count: 6,346

Key words: Readiness Potential; Self-initiated action; Prediction; Uncertainty; Learning

Open Science: All of the materials used in this study, the data, and the analysis scripts are available to download at https://osf.io/menvj/

Contact: Dr Eoin Travers (e.travers@ucl.ac.uk), UCL Institute of Cognitive Neuroscience, 17 Queens Square, London WC1N 3AZ, UK 


\section{Introduction}

Human actions are guided by a combination of external cues, internal intentions and stored knowledge. Self-initiated voluntary actions - those produced without any immediate external prompt - are preceded by a slow EEG Readiness Potential (RP) that ramps up over the second or so prior to action (Deecke et al., 1969). The RP is primarily generated by the Supplementary and preSupplementary Motor Areas (SMA and pre-SMA; Shibasaki \& Hallett, 2006), which in turn receive strong drive from the subcortical circuitry of the basal ganglia. Interestingly, the readiness potential is reduced or absent prior to externally-triggered actions that are temporally matched to each participant's voluntary actions (Jenkins et al., 2000). This result suggests that it is a specific neural correlate of voluntary action.

Why does the RP occur, and what does it represent? Accounts of the RP tend to focus on one of two contrasting facets of self-initiated actions. Some accounts emphasise the random, unconstrained, and unpredictable nature of self-initiated actions (Eccles, 1985; Jo, Hinterberger, Wittmann, Borghardt, \& Schmidt, 2013; Nachev, Rees, Parton, Kennard, \& Husain, 2005; Schurger, Sitt, \& Dehaene, 2012). Others emphasise the unique role of planning and temporal expectations in internally-generated actions (Brunia, Boxtel, \& Böcker, 2011; Verleger, Haake, Baur, \& Śmigasiewicz, 2016).

\section{Randomness and Uncertainty in Action}

Schurger and colleagues (Erra, Arbotto, \& Schurger, 2019; Schurger, Sitt, \& Dehaene, 2012; see

20 also Murakami, Shteingart, Loewenstein, \& Mainen, 2017) recently proposed that the timing of self-initiated actions may effectively be random. In this model, actions are triggered when randomly fluctuating neural activity reaches a threshold. When this activity is time-locked to action and averaged across trials - the usual way of analysing EEG from RP experiments - this model reproduces the classic time course of the RP. We will refer to this account as the stochastic model. The RP usually begins a second or more prior to action (Shibasaki \& Hallett, 2006). When participants are asked when they decided to move, they typically indicate a time just moments prior to action (Libet, 1985), leading to the interesting conclusion that voluntary actions must have unconscious causes (Libet et al, 1983). The stochastic model explains this apparent contradiction. It holds that there is no specific neural event corresponding to the start of the RP. Rather, the apparent "onset" of the RP is merely an artefact of averaging across trials. If any decision to move exists, it would necessarily occur after a stochastic neural signal reached some predefined threshold for action. 
Could self-initiated actions really be random? Random actions can sometimes be useful (Glimcher, 2005; Maye et al., 2007). In animals, the ability to produce actions that cannot be predicted by prey or by predators can have an adaptive value (Brembs, 2011; Maye et al., 2007; Maynard Smith, 1982). Randomness also plays an important role in optimal models of decisionmaking in uncertain environments. In a familiar environment, the optimal policy is to consistently choose whatever action has the best pay-off. In an uncertain environment, one must strike a balance between exploiting immediately available options and exploring potentially better alternatives

40 (March, 1991). A common way to achieve this is through stochastic action selection: usually choosing what one believes to be the best option, but sometimes randomly exploring other options instead (Gershman, 2018). The Thompson sampling algorithm (Thompson, 1933), where the degree of randomness is proportional to the agent's uncertainty about which action is best, is close to the optimal policy in many environments, and approximates human behaviour (Gershman, 2018).

Nachev and colleagues (Nachev et al., 2005, 2008) proposed a slightly different view. They argued that SMA and pre-SMA activity, and hence presumably the RP, reflects conflict or uncertainty due to the lack of external constraint on self-initiated actions. In simple self-initiated actions, conflict occurs because there are many possible times at which one could act, but no reason to favour one over the others - the decision to act is underdetermined. Nachev et al. (2008) therefore suggest that medial frontal activity, as measured by RP, may reflect uncertainty about when to act, just as the anterior cingulate, located just ventral to SMA, reflects uncertainty about which action to perform (Botvinick et al., 2004). This account, like the stochastic account, predicts a greater RP when the reasons for action are least clear or most uncertain.

Zapparoli et al. (2018; see also Seghezzi et al., 2019) report MRI results consistent with this idea. They asked participants to perform actions in response to cues, or to freely decide either what action to perform, when to act, and whether or not to go ahead with the action (Brass \& Haggard, 2008). They found stronger SMA activation for free actions than cued actions, and found that SMA activity was stronger for free decisions about when to act than for free decisions about what action to perform, or whether to act.

\section{Planning, Temporal Expectation, and Action}

Other explanations link the RP not to randomness, but to determination and imposition of structure and pattern on human behaviour. These views emphasise the unique role of planning and temporal expectation in self-initiated actions. The sensory consequences of a voluntary action are thought to be automatically and unconsciously predicted by a forward model (Blakemore et al., 2002). Thus, self-initiated actions are events that the actor expects to occur (Friston et al., 2010), and which 
represent a reduction in entropy, or the imposition of an internal model on the external world (Parr \& Friston, 2019). This has two implications. First, an agent can begin to prepare an action long before they intend to perform it. Second, they can have expectations about when an action and its consequences will occur. The RP could reflect either of these processes.

Voluntary movements must be prepared before they are executed (Lara et al., 2018; Wise, 1985). Direct motor and premotor cortical recording from monkeys and rodents (e.g. Elsayed, Lara, Kaufman, Churchland, \& Cunningham, 2016; Lara et al., 2018; Murakami et al., 2017) show that motor preparation involves passing through a sequence of neural states. Similar preparatory states may occur prior to self-initiated actions and prior to speeded, externally-triggered actions, but preparation of self-paced actions often takes more time (Lara et al., 2018). The RP reflects the firing rates of populations of motor and supplementary motor neurons (Shibasaki \& Hallett, 2006). Therefore, it provides a one-dimensional readout of the multidimensional pre-movement neural activity captured by direct cortical recordings. This implies that the RP may not be a distinctive feature of self-initiated actions. Instead, the RP could reflect a general process of motor preparation, which can either be performed rapidly in response to an imperative stimulus, or, if necessary, initiated endogenously, and extended over a long period of time.

There is also a strong link between motor preparation and temporal expectation. People often prepare in advance to ensure they can act at the right time. The medial frontal cortex, including SMA, is also involved in temporal anticipation of future events (Tecce, 1972) and in processing the passage of time (Kolling \& O'Reilly, 2018). The RP is strikingly similar in topography and timing to the Contingent Negative Variation (CNV; Brunia et al., 2011; Tecce, 1972), a slow negative component that ramps up prior to the time at which participants expect to receive a behaviourally relevant stimulus. $\mathrm{CNV}$ is typically recorded in reaction time experiments where a warning cue occurs at a fixed interval prior to a "Go" signal. A number of authors have

90 argued that the RP and CNV both reflect slow motor preparation (Brunia et al., 2011; Grünewald et al., 1979; Rohrbaugh \& Gaillard, 1983): the RP represents preparation of a self-timed action, while CNV represents preparation of an action timed to a predictable external event. This conclusion is supported by source localisation of CNV to supplementary motor areas (Hultin et al., 1996). Interestingly, a smaller CNV occurs even when participants are not required to respond to the stimulus, suggesting that it also reflects non-motor temporal processes (Rohrbaugh \& Gaillard, 1983). The RP may also occur in the absence of immediate action, since it is apparently also found prior to "covert decisions" that do not immediately lead to actions (Alexander et al., 2016; Gluth et 
al., 2013). These findings indicate that the RP and CNV may reflect both motor preparation and temporal expectation.

The contribution of temporal expectation to the RP is supported by classical findings. Libet and colleagues (Libet, 1985; Libet et al., 1983) distinguished between Type I and Type II RPs (see also Frith \& Haggard, 2018). When participants reported that they had preplanned in advance the time at which they would move, RPs began early and reached a high amplitude (Type II RPs). When participants instead acted "freely and capriciously" (Libet et al., 1983) without extensive preplanning, RPs began just before action, and reached lower amplitudes (Type I RPs). However, the distinction between Type I and Type II RPs was made on the basis of participants' subjective reports about their general strategy for initiating actions. These reports were apparently made at the end of a block of several trials, so cannot be readily linked to individual action events. Other studies (e.g. Schurger, 2018) have noted that the RP tends to be greater on trials where participants

110 spontaneously waited longer than usual before acting. Similarly, Jenkins et al. (2000) showed that SMA activity (recorded using PET) is high for both self-paced actions and responses to a predictably-timed cue, but reduced for responses to an unpredictable cue.

\section{Randomness or Planning?}

In most studies of the RP, both planning and expectation, and randomness and uncertainty, might all play a role in action. Participants may produce actions that are unconstrained by external stimuli, and so appear random and unpredictable to an observer. Nonetheless, these actions might well be preplanned, and so the movement and its consequences may be expected by the participant themselves. In other words, random behaviour might in fact be carefully planned.

As a result, these descriptive studies cannot determine whether the RP reflects a process of planning and predetermination on the one hand, or randomness, uncertainty and lack of determination on the other. To separate these two possible influences, we need an experimental design which explicitly manipulates the degree of determination/indeterminacy of action, and investigates how this affects the RP. Here we do this by providing a context where participants must learn, through trial and error, the optimal time to act. This design encourages gradual acquisition of preparatory planning for action, as participants learn when they should act. The task thus encourages a progressive learning-related shift from "capricious", random behaviour to regular, preplanned behaviour (Libet, 1985). Our design would initially encourage random exploration of the environment, for example, by acting at various different times, and monitoring

130 the outcome. These early actions are unpredictable and unconstrained by the environment. While 
such exploratory actions might in principle be preplanned, preplanning them offers no obvious advantage over simply relying on a random generator. Later, after becoming more certain about when they should act, participants should consistently wait a fixed time before doing so. These later, post-learning actions would be strongly constrained by the environment, or at least by the historical environment of previous actions and outcomes. There is an obvious advantage to preplanning them, and an obvious disadvantage to allowing their timing to vary randomly. Therefore, learning the optimal time to act offers an experimentally-tractable way to investigate whether the RP reflects the process of determining an action, or rather represents the indeterminacy and randomness of action generation (Figure 1). If the RP reflects randomness and absence of constraint, RPs should decrease over time as participants progress from random exploration towards planning based on an internal model of the optimal time interval. If the RP instead reflects planning, RPs should increase over with learning (if we assume that initial exploratory actions are not preplanned but late actions are preplanned) or should at least stay constant (if we assume that even initial exploratory actions in fact derive from strategic plans to sample across the distribution of action times).
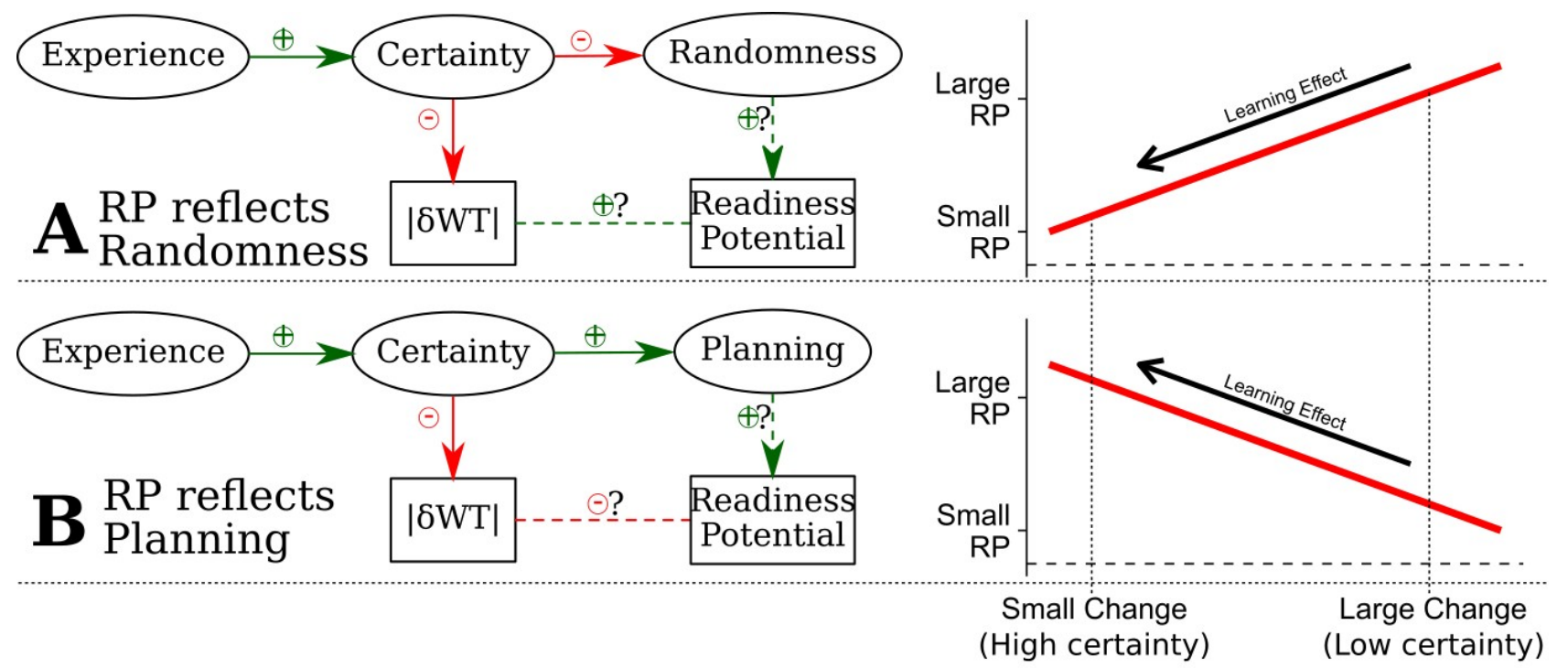

Change in Wait Time $(|\delta W T|)$

Figure 1. Predictions. Ovals show latent variables, rectangles observed variables, and arrows causal effects. Question marks indicate hypothesised relationships. As participants gain experience over the course of each block, their certainty in the correct time to act increases, trial-to-trial changes in their waiting times $(|\delta W T|)$ decrease, planning, certainty and temporal expectation increase, and the role of randomness or stochasticity and uncertainty in action timing decreases. $\boldsymbol{A}$. If randomness or uncertainty drive the $R P$, it will have greatest amplitude on trials with large 
changes in waiting times (positive relationship between $|\delta W T|$ and RP magnitude). B. If planning drives the RP, it will be greatest on trials with small changes in waiting times (negative relationship between $|\delta W T|$ and RP magnitude). Since our learning task required reduction of temporal uncertainty, we show the effects of learning not as a function of time or trial number, but as our behavioural proxy for (un)certainty, namely the trial-to-trial update in action time $(|\delta W T|)$ that indicates progressive acquisition of the to-be-learned optimal time of action. Black arrows indicating the progress of learning over time thus run, unusually, from right to left.

\section{Method}

\section{Participants}

We aimed to test 20 participants, based on the sample size used for previous RP studies (e.g. Khalighinejad et al., 2018). Twenty-one healthy participants (11 female, mean age \pm SD: $24.24 \pm$ 4.37 years) took part in the experiment. We excluded two participants who moved excessively during EEG recording. Thus, 19 participants were included in the analyses (10 female, mean age \pm SD: $23.53 \pm 3.58$ years). Participants received $£ 7.50$ per hour reimbursement upon completing the experiment, plus a bonus for performance on the task. The experiment was approved by the UCL ICN ethics committee and each participant's written informed consent was collected before starting the experiment.

\section{Procedure}

We developed a temporal reinforcement learning paradigm that allows participants to learn, through experience, the best time to act. Our cover story treated participants as bakers, placing a soufflé in an oven at the beginning of each trial. Their task was to wait until the soufflé was ready, and then press a key to withdraw it from the oven to gain a small cash bonus. If participants withdrew a soufflé before it was ready, that trial was aborted and the next began after a short interval. Thus, participants learned the optimal baking/waiting time through this action feedback. Crucially, the average baking/waiting time required varied between blocks, and had to be learned from feedback.

Participants completed 15 blocks in total, taking 3 minutes each, and were instructed to score as many points as possible in that time. Soufflés were ready after an average of 3, 5, 7, 9, or 11 seconds depending on the block, with a SD of 1 second across trials in each block. There was no explicit penalty for leaving the soufflé too long, but doing so reduced the number of trials a participant could complete in the time available. The colour of the oven changed with each new block to provide a salient visual cue indicating a change in optimal waiting times. 
Before starting the experiment, participants were shown two sample trials by the experimenter. Following this, participants completed one three-minute round in order to get familiar with the task and to check their understanding. The baking time of soufflés in the trial round was normally distributed with a standard deviation of 1 and a mean of 7 , giving the participants a reasonable prior expectation for the real task.

If participants knew the distribution of baking times for each block, the optimal strategy would be to wait for time $\tau$ when they are sufficiently certain that the soufflé was ready before withdrawing it. Since they had to learn this information, the optimal strategy is to initially explore the effects of opening the oven (that is, acting) after different times to estimate $\tau$, and then to use that estimate to score as many points as possible. This can be achieved through Bayesian learning strategies such as Thompson sampling. An agent using such a strategy begins each block with low posterior precision in their estimate of $\tau$. They will thus be highly stochastic in their actions, but learn quickly from new evidence. As they learn, their estimate becomes more precise, their actions less stochastic, and their response to new evidence reduced.

\section{EEG Acquisition}

The experiment was conducted in an electrically shielded room. We used a BioSemi ActiveTwo system (BioSemi, 2011) to record 32-channel EEG. Electrodes were placed in locations FP1, FP2, F7, F3, Fz, F4, F8, FC5, FC1, FCz, FC2, FC6, T7, C3, C1, Cz, C2, C4, T8, CP5, CP1, CPz, CP2, CP6, P7, P3, Pz, P4, P8, O1, Oz, and O2. This montage includes a higher than usual density of electrodes clustered around $\mathrm{Cz}$ to capture motor activity. Electro-oculogram (EOG) was recorded with electrodes above and below the right eye and on the outer canthi of both eyes to control for eye movement artefacts. EEG was sampled at $500 \mathrm{~Hz}$.

\section{EEG Preprocessing}

EEG data preprocessing was performed with Python using the MNE software package (Gramfort et al., 2013). Data were bandpass filtered between 0.1 and $250 \mathrm{~Hz}$, and downsampled to $250 \mathrm{~Hz}$ for analysis. As electrodes were more densely clustered around $\mathrm{Cz}$, a pure average reference would disproportionately subtract activity from this area. Ideally, an average reference signal should give equal weights to signals from all areas of the scalp. Therefore, we used the average of all electrodes excluding FCz, C1, C2, CPz, T7, and T8 as a more representative reference signal. Data with large motor artefacts were removed by visual inspection. Independent component analysis (ICA) was then used to identify eye movement and blink artefacts from the EEG data. Eye-movement related 
ICA components were identified by visual inspection and by correlating their activity with that of the EOG channels.

RP epochs were extracted from 3 seconds before to 0.5 seconds after action. Epoch

220 recordings were baseline-corrected to the average of the window 3 to 2.9 seconds prior to action.

Trials with maximum EEG amplitudes $>2 \sigma$ from the mean (where $\sigma$ was estimated using a robust tdistribution; see Supplementary Materials) were excluded, comprising 15.8\% of the data. To reduce noise for single-trial analyses, we averaged the signal from the five electrodes around location FCz: $\mathrm{Fz}, \mathrm{FC} 1, \mathrm{FCz}, \mathrm{FC} 2$, and $\mathrm{Cz}$. To quantify the RP amplitude on a single trial, we compared the average voltage on these electrodes in the final $50 \mathrm{~ms}$ prior to action, to a baseline defined as the average signal between 3 and 2.9 s prior to action (Figure 3B). Cases where participants waited less than 2 seconds before responding $(0.7 \%$ of trials) were excluded from further analyses. 

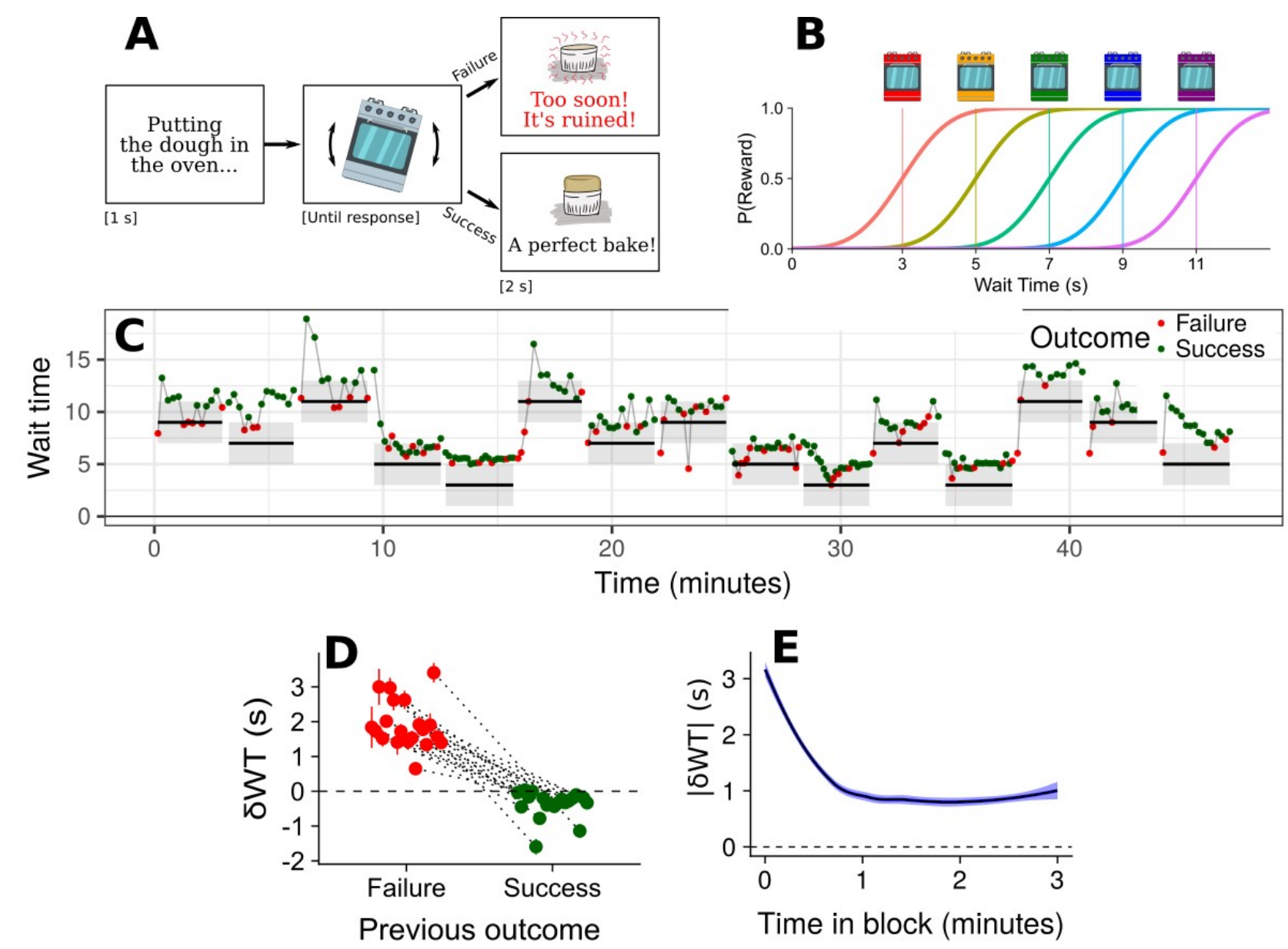

Figure 2. Temporal reinforcement-learning task and behavioural results. A. Outline of behavioural task. A soufflé was placed in the oven at the start of each trial, and participants had to wait an unknown time before pressing a button to withdraw it. Points were awarded only on trials where action was not premature. $\boldsymbol{B}$. The time participants needed to wait before acting differed across conditions (different coloured ovens), and had to be learned through experience. $\boldsymbol{C}$. Waiting times across the experiment for a single participant. Horizontal bars show mean $\pm 2 S D$ times at which soufflés were ready in each block. Green (red) dots show trials where participants did (not) wait long enough. D. Participants waited considerably longer after trials where they acted too soon (failures) and acted slightly sooner after a previous trial where they waited long enough to retrieve the soufflé (successes). Error bars show standard errors for each participant. E. Trial-to-trial changes in waiting times decreased over the course of each block. Thus, people learned a better time for action. Error bars show lowess standard error, pooling data from all participants. 


\section{Results}

\section{Behavioural results}

Participants' behaviour is consistent with a simple iterative reinforcement learning strategy (Figure 2C-E). If their action was too early (i.e., feedback showed soufflé not yet ready) on trial $t-1$, participants waited $1.9 \mathrm{~s}$ longer $(\mathrm{SD}=0.7 \mathrm{~s})$ on trial $\mathrm{t}, \mathrm{t}(19)=12.63, \mathrm{p}<.001$, one-sample t-test against zero. If their action was later than required (i.e., feedback confirmed that soufflé was already baked on trial $t-1)$, participants acted $0.4 \mathrm{~s}(\mathrm{SD}=0.4 \mathrm{~s})$ earlier on trial $\mathrm{t}, \mathrm{t}(19)=4.28, \mathrm{p}$ $250<.001$.

The amount participants adjusted their wait times in response to feedback decreased progressively in each block (Figure 2C), indicating strong learning initially, and weaker learning later on. By reducing their learning rates in this way, participants can home in on the optimal waiting time in a way that approximates Bayesian learning. In other words, participants should, and do, substantially update how long they wait before acting in response to feedback early in learning, when they are uncertain of the correct time. Conversely, later in learning they should update their wait times only slightly, because they have acquired greater certainty about the optimal time to wait. We used absolute, unsigned changes in participants' wait time from one trial to the next, | $\delta \mathrm{WT} \mid$, as a measure of their uncertainty about the optimal time to act, and so of how exploratory or stochastic their actions were. This lets us characterize individual responses as being more exploratory (strongly different from time of previous action, therefore high $|\delta \mathrm{WT}|$ ) or more exploitative (repeating time of action from previous trial, therefore low $|\delta \mathrm{WT}|$ ).

We used $|\delta \mathrm{WT}|$ rather than trial number, or progress through a block, as our proxy for certainty in later analyses for several reasons (see also Figure 1). First, the number of trials a participant completes per block depends on how long they wait on each trial. Trial counts therefore vary considerably between participants and between blocks, and trial number cannot readily be compared across blocks. Second, the time and number of trials needed to converge on a consistent waiting time varies between participants and blocks, so that a participant might be very certain about when to act after one minute, or after 10 trials, on one block, but be unsure at the same point

270 in time, or after the same number of trials, in another block. Since $|\delta \mathrm{WT}|$ is a consequence of participants' level of certainty, which is the focus of the learning task, it avoids these ambiguities. Further, on some trials participants updated their waiting times in the wrong direction, acting later after success or sooner after premature responses. We assume that the absolute magnitude of the update indicates that they were uncertain. In contrast, the sign of the update may simply indicate 
whether they responded appropriately to their own uncertainty. We therefore used the absolute magnitude of the update in waiting time in either direction as our measure of certainty. A small update in either direction is taken to reflect high certainty about when to act, and a large update is taken to reflect high uncertainty.

\section{EEG Analysis}

280 To test the effect of uncertainty while controlling for possible confounds, we fit a linear mixed model with single-trial RP voltage as the dependent variable, $|\delta \mathrm{WT}|$ as our predictor of interest, and signed $\delta \mathrm{WT}, \mathrm{WT}$, and block number as covariates. Controlling for block number allows us to rule out the possibility that any effects are due to changes in RP amplitude over the course of the experiment. We do not control for trial number within each block, as this measure is highly collinear with $|\delta \mathrm{WT}|$. All parameters were allowed to vary between participants as random effects. We initially calculated (restricted) maximum-likelihood parameter estimates using the lme4 package for R (Bates et al., 2014), but found that the optimizer failed to converge on the random effect estimates. We therefore used the brms package (Bürkner, 2017) to perform Bayesian estimation, avoiding these convergence issues. We used uniform priors on the fixed effects, and

290 obtained estimates and credible intervals that matched the maximum-likelihood parameters to one decimal place, along with more robust estimates of the random effects. We therefore report random effects estimates from the Bayesian model, fixed effect estimates which were the same in both models, and fixed effect p-values from the maximum-likelihood model for hypothesis testing. Full details of the Bayesian and maximum-likelihood models can be found in supplementary materials. For all effects, we report unstandardised regression weights $b, 95 \%$ confidence/credible intervals, and estimated standard deviation of the effect across participants. For visualisation purposes (Figure $3 \mathrm{C})$, we coded trials as being either above the median value of $|\delta \mathrm{WT}|$ for that block (relatively exploratory responses) or below it (relatively exploitative responses). Since $|\delta \mathrm{WT}|$ is compared to the median value within each particular block, half of the trials in each block are coded as exploratory, half as exploitative. This eliminates any confounds due to systematic differences between blocks.

\section{EEG Results}

A clear RP occurred prior to participants' responses in this task (Figure 3A-B). Average RP ERPs for explore and exploit responses are plotted in Figure 3C. The RP appears to have a greater amplitude for exploit actions (low $|\delta \mathrm{WT}|$ ) than explore actions (high $|\delta \mathrm{WT}|$ ) throughout most of its time course. This was confirmed by the linear mixed model analysis on RP amplitudes in the final 
phase before action. Thus, there was a significant effect of $|\delta \mathrm{WT}|$ (in seconds) on RP amplitudes (in $\mu \mathrm{V}), b=-0.29 \mathrm{CI}=[0.02,0.56], \mathrm{p}=.038, \mathrm{SD}$ across subjects $=0.16$, meaning that greater trial-totrial updates in waiting times were associated with lower (less negative) RPs. This is consistent with

310 the averaged ERP results. There was no effect of signed $\delta \mathrm{WT}$ (increasing or decreasing wait times), $b=0.04, \mathrm{CI}=[-0.19,0.27], \mathrm{p}=.760, \mathrm{SD}$ across subjects $=0.16$, of actual wait time (longer or shorter wait times), $b=-0.03 \mathrm{CI}=[-0.20,0.15], \mathrm{p}=.782$, SD across subjects $=0.27$, or of block number, $b=-0.01 \mathrm{CI}=[-0.09,0.06], \mathrm{p}=.751$, SD across subjects $=0.08$. 

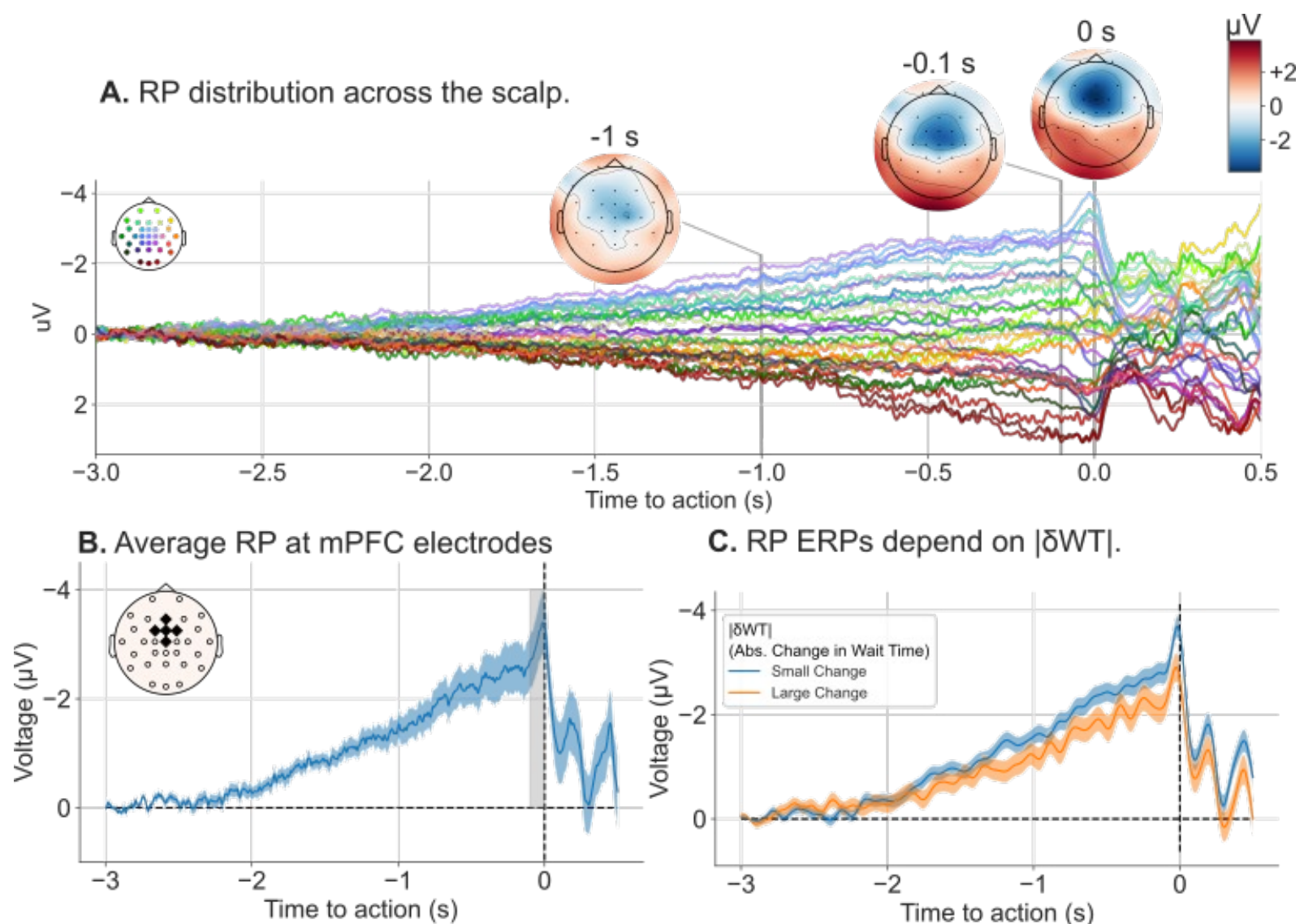

C. RP ERPs depend on $|\delta W T|$.

D. Single-trial RP amplitudes depend on $|\delta W T|$

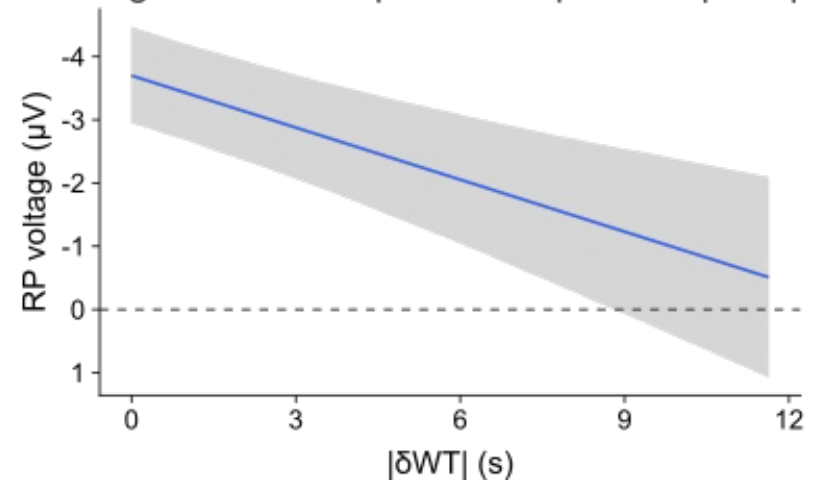

Figure 3. EEG Results. A. A clear Readiness Potential (RP) occurred prior to action. B. RP EventRelated-Potential (ERP) averaged across centro-frontal electrodes ( $F z, F C 1, F C z, F C 2$, and $C z$ ). The average voltage in the last $50 \mathrm{~ms}$ prior to action (grey) was used as an estimate of single-trial $R P$ voltages. $C . R P$ ERPs for actions where absolute changes in wait times $(|\delta W T|)$ were larger (orange) or smaller (blue) than the median for that block, respectively indicating relatively exploratory or relatively exploitative actions. D. Estimate $( \pm S E)$ of single-trial RP voltages as a function of $|\delta W T|$, after adjusting for other terms in the model. 


\section{Discussion}

Does the Readiness Potential reflect randomness and uncertainty, or planning and expectation? We found that RP amplitude increases as participants learn through experience how long to wait before acting, so that their certainty about action time increases, and their actions become less random, more preplanned and more predictable. This is consistent with the proposal that the RP reflects anticipatory planning and preparation to make an action at a specific time in the future. The link between RP and planning of course is not new, and may seem intrinsic to the classical concepts of willed action and self-paced action (Kornhuber \& Deecke, 1965). Libet et al. (1983) noted that RPs started earlier, and reached higher amplitude when participants reported preplanning voluntary actions, compared to when they did not. However, the method used to elicit these self-reports is unclear, and does not allow individual motor acts to be classified as more planned, or less planned (Frith \& Haggard, 2018). In contrast, our work operationalises planning as the acquisition of an internal model of an optimal time to act. We consider the link between a classic parameter of learning models, namely the behavioural update, and the neural signals preceding voluntary action. We show that RP amplitude is positively associated with deploying a plan for action based on progressive learning of an internal model. Our finding thus provides an interesting neuropsychological link between volition and the general idea of model-based behaviour. It should be noted, however, that the effect of $|\delta \mathrm{WT}|$ on the amplitude of the RP, our main result, is not particularly large. On average, a $1 \mathrm{~s}$ increase in $|\delta \mathrm{WT}|$ led to a $0.28 \mu \mathrm{V}$ decrease in RP amplitude, while the average RP amplitude was $\sim 3 \mu \mathrm{V}$.

Recordings from frontal and prefrontal cortical neurons in animals have also informed neuroscientific theories of planned behaviour (Rushworth et al., 2004). For example, monkeys can learn a sequence consisting of multiple successive movements, performed in strict order. While previous discussions focussed on representation of sequence complexity, some results recall the planning of simple actions we have studied here. When the animal performs sequences of movements from memory, some neurons in medial frontal cortex gradually increase their firing rates either before the sequence begins, with a profile resembling the RP (Tanji \& Shima, 1994), while others fire during the interval between one movement and another. The animal learns, through extensive training, a representation of what to do, and when to do it, at the level of both overall sequence, and individual movement. Our results show medial frontal activity in humans may reflect the progressive acquisition and deployment of such a representation to guide action choice. It appears inconsistent, however, with the proposal that the RP reflects uncertainty (Nachev et al., 2008), or arises as the result of a purely stochastic triggering process (Schurger et al., 2012). 
Our findings are based on the relationship between trial-by-trial changes in waiting times, which are due to learning, and the amplitude of the RP on individual trials. We did not include control blocks where feedback was given at random, so that participants could not learn the optimal waiting time. Thus, we cannot completely exclude the possibility that RP amplitude simply increases over time. However, our pilot experiments showed that including these random-feedback blocks prevented some participants from learning from true feedback on subsequent blocks. Fortunately, there are a number of reasons to believe the increase in the RP is due to experience, and not due to the passage of time. First, evoked EEG potentials typically habituate, or become smaller with repetition (Groves \& Thompson, 1970). This is also true of the CNV (Kononowicz \& Van Rijn, 2011), a near relative of the RP. We are not aware of previous studies examining whether the RP habituates. Habituation would predict that the RP should decrease progressively during each block, whereas we observed an increase. Second, participants moved to a new block every three minutes. If the increase in RP amplitude was due to the passage of time, we would expect to see greater RPs in later blocks. However, we included block number as a predictor in our analyses, and found no relationship, $b=-0.01 \mathrm{CI}=[-0.09,0.06]$.

\section{Accumulator Models of Action}

Schurger et al. (2012) proposed that spontaneous self-initiated actions could be triggered by a neural circuit that accumulates random noise until it reaches a threshold. They showed that the RP could reflect random fluctuations in accumulated noise, time-locked to the time they crossed threshold. How might such a model apply to our paradigm?

One possibility is that the Schurger et al. (2012) model applies only to pure self-initiated actions where timing is unspecified, and not to actions during model-based cognitive tasks such as ours, where there is an optimal time to act. However, there was a clear RP prior to action in our task

380 (Figure 3A). If this model does not apply here, we must conclude that it is not an explanation of the $\mathrm{RP}$ in general, but rather an explanation of the RP under specific condition that participants are asked to act, but given no guidance at all about when to act.

Second, actions might be generated by several different pathways, only some of which give rise to an RP. Thus, the lateral premotor pathway for externally-triggered actions has been distinguished from the medial frontal pathway, based on pre-SMA and SMA, for internallytriggered actions (Passingham, 1993). Similarly, there might be one mechanism for generating spontaneous, arbitrary actions, and one for deliberate, preplanned movements. Our results might arise because the latter generates stronger RPs than the former. This view recalls Libet et al.'s 
(1983) distinction between Type I and Type II RPs (see above). An analogous distinction was proposed by Maoz et al. (2019), who reported a smaller RP prior to responses to value-based decisions (choosing to donate to one of two possible charities) than prior to arbitrary actions (pressing one of two buttons at random). Those authors suggested that arbitrary actions are triggered by the accumulation of noise in SMA (Schurger et al., 2012), leading to an RP, while value-based actions are triggered by another mechanism, possibly in ventromedial prefrontal cortex, that does not lead to an RP (Wallis, 2007).

In most contexts, including our task, actions are neither purely random nor purely valuebased, but are hybrid actions driven by some mixture of these processes (Luce, 1959). Any particular action might be triggered by just one of these mechanisms (e.g., Obhi \& Haggard, 2004), or by a combination of the two (see Hughes et al., 2011). In any event, the random mechanism may 400 be assumed to play a greater role earlier compared to later in learning, while the converse holds for the value-based mechanism. If the stochastic accumulation mechanism is the generator of the RP, we would expect to find an RP of greater amplitude early in learning, where randomness would be a functional part of exploration strategy. Since we found the opposite effect, our results are inconsistent with the interpretation of the RP as reflecting a random process for triggering actions, at least in the context of the present task.

A third possibility is that the same neural accumulation mechanism is responsible for the timing of both random, arbitrary actions and planned, value-based actions, but that the input to the accumulator differs. Arbitrary actions could be triggered by random noise in the accumulator, while value-based actions could be triggered by a specific external forcing input. We have simulated this 410 possibility. Briefly, the RP can indeed be reproduced by a model that accumulates random noise in the absence of specific inputs. However, we find that the same model predicts greater RP amplitudes when driven by clear evidence that one should act now, via a forcing input. We illustrate this idea in Figure 4, and describe the simulations used to reach it below. The full simulation parameters and the python code used to conduct the simulations can be found in Supplementary Materials.

In our simulations we assume that the input $I_{t}$ to the accumulator over time depends on the agents' posterior belief that an action at that time would be rewarded. Since the distribution of reward times is Gaussian, the posterior estimate of $\mathrm{P}$ (Reward|Act Now), marginalising over possible readiness times $\mu$, is a cumulative Gaussian function centred around $\hat{\mu}$, an estimate of the 420 average time taken for a soufflé to be ready, with a slope $\beta$ that increases as the estimate of $\hat{\mu}$ becomes more precise: $I_{t}=\Phi(\beta(\mathrm{t}-\hat{\mu})$, where $\Phi$ is the cumulative Gaussian function. Prior to any 
experience, uncertainty about $\hat{\mu}$ is extremely high, so $\beta$ is close to 0 , the forcing input to the accumulator is zero or weak, and moreover is constant over time. Therefore, the timing of action is largely determined by random noise in the accumulator (Figure 4A). This is equivalent to the model proposed by Schurger et al. (2012). With learning, the estimate of $\hat{\mu}$ becomes more precise, the input to the accumulator becomes more temporally specific, and actions come to be triggered directly by this step-like forcing input (Figure 4B-C). As a result, simulated wait times cluster more closely around a time shortly after $\hat{\mu}$. 
A. Noise only

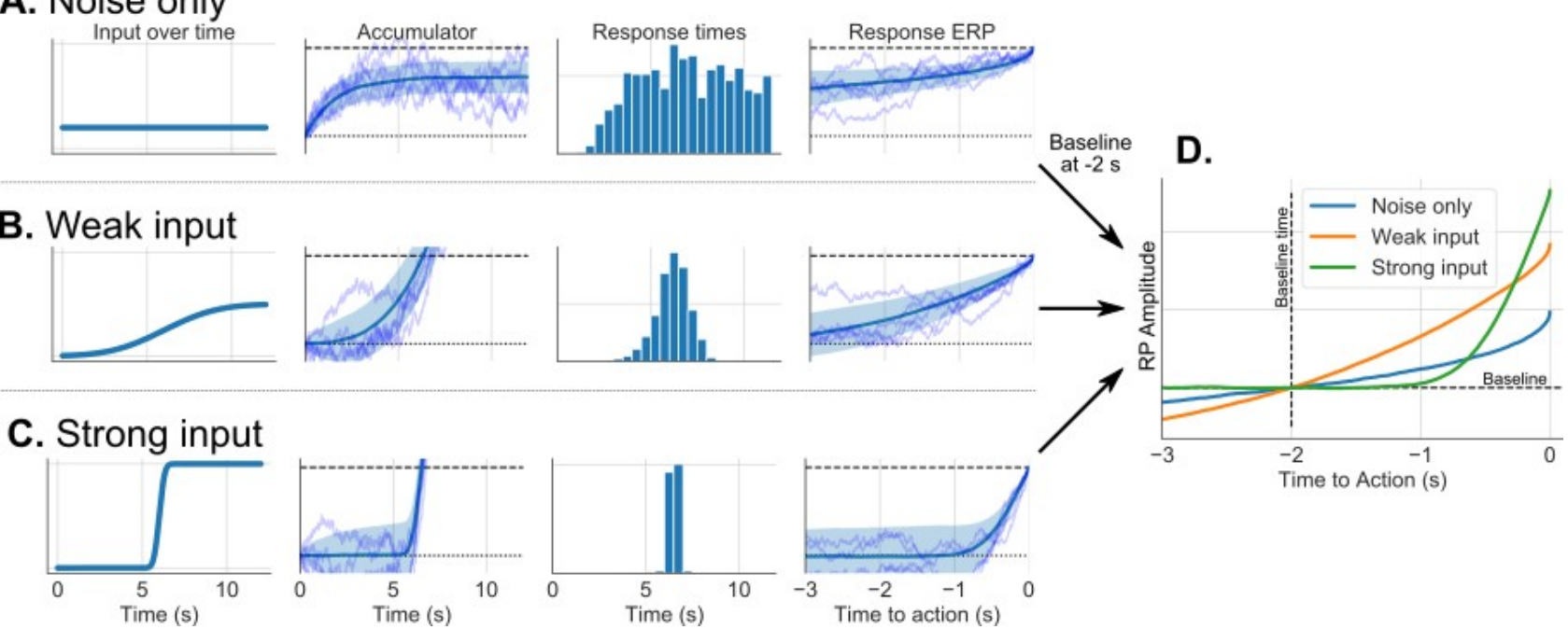

430

Figure 4. Simulation results from a model where an input signal (first column) is accumulated over time with noise (second column). The model predicts both response time distributions (third column) and RP shape (final column) A.) the observer is highly uncertain of the correct time to act (constant weak input), B.) the observer is somewhat uncertain of the correct time to act (weak gradually increasing input). $\boldsymbol{C}$.) the observer is highly certain of the correct time to act (strong, temporally precise input. D. Simulated RP amplitudes, baseline-corrected $2 \mathrm{~s}$ before action. Although the threshold for action is the same, differences in activity at the (arbitrarily-chosen) baseline time mean that RP amplitudes appear greatest when action is triggered by a strong input signal (as in C), and weakest when triggered by stochastic noise only (as in A).

What happens to the RP? In these models, actions are triggered once the accumulator reaches a fixed threshold. This means that the level of the accumulator at the time of action is the same in all cases. However, the state of the accumulator during the baseline window a few seconds prior to action can change across simulations, and these changes can account for differences in the apparent amplitude of the RP. Therefore, we will focus on the shape of the simulated RP, and the time at which it appears to begin rising to threshold. When actions are driven by noise (Figure 4A), the simulated RP obtained by time-locking accumulator traces to the simulated response times reproduces the classic shape of the RP (Schurger et al, 2012). Since the shape of the RP here is an artefact of biased sampling of random fluctuations, there is no specific moment at which the RP

450 begins. Instead, the slope is steepest close to the time of action, and appears progressively shallower further back in time. This also means that the accumulator is already moderately activated $2 \mathrm{~s}$ prior 
to action. As a result, the amplitude of the $\mathrm{RP}-$ the change in activity from $-2 \mathrm{~s}$ to the time of action - is small here.

As participants become more certain of the optimal time of action (Figure 4B), there is a clear input to the accumulator prior to actions, and the simulated RPs have a clearer onset time. Later still, when the correct time to act is precisely known (Figure 4C), the input to the model ramps up sharply at this time, and the accumulator quickly rises to threshold. From these simulations, we can see that the apparent amplitude of the RP depends not on the state of the accumulator at the time of action, but on how much the accumulator changes between the baseline and the time of

460 action. As a result, the apparent RP amplitude is largest when actions are triggered by a strong input signal, and smallest when triggered by stochastic fluctuations in the accumulator. Thus, if the RP wholly or partly reflects stochastic fluctuations in an evidence accumulation process, then increasing the contribution of the stochastic process does not necessarily produce a larger RP. Rather, greater stochasticity leads to a slower, more gradual rise of the RP.

These results highlight a fundamental limitation of EEG and related approaches. These recordings allow us to infer changes in neural activity over time, but not absolute firing rates. Thus, we can only estimate the amount of activity at the time of action relative to a pre-action baseline, and not the actual level of activity at the time of action. We can try to reduce the risk of distorting apparent RP amplitudes by using a baseline window long before action and a longer ERP.

470 Unfortunately, EEG data contains high-amplitude, low-frequency noise components, and the highpass filter used to attenuate this noise would also distort longer ERPs. These issues are avoided by direct electrophysiological recordings, although these are only rarely possible in humans. Notably, direct recordings in macaques (Lara et al, 2018) show that the same patterns of firing rates occur prior to both self-initiated and externally-triggered actions. This finding is consistent with a constant accumulator threshold for action.

\section{Readiness Potentials and Anticipation}

An important limitation of these accumulator models is that they do not capture the kind of temporal expectation and anticipation that some claim the RP reflects (e.g. Brunia et al., 2011). In an accumulator model the state of the accumulator will either ramp up quickly if there is a strong 480 external signal, or ramp up slowly if the external signal is weak or absent. In contrast, temporal expectation can be captured in predictive processing models of cognition and motor control (Blakemore et al., 2002; Wolpert et al., 2003). These models are commonly used to explain the consequences of self-initiated actions (Farrer \& Frith, 2002; Haggard, 2017), but do not generally 
focus on the precursors of actions, nor on temporal dynamics in the seconds prior to action. Thus, they cannot readily provide a mechanistic explanation of preparation and RP. A challenge for future work will be to reconcile evidence accumulation and predictive processing accounts. To do this, it will be necessary to develop models that capture the temporal dynamics of evidence accumulation both during action preparation and during action outcome representation.

Some authors argue that RP and CNV both reflect a single underlying process of temporal anticipation and motor preparation (Brunia et al., 2011; Rohrbaugh \& Gaillard, 1983). Our results are consistent with this theory. However, another interpretation is possible. It might be that the RP reflects spontaneity and uncertainty in self-generated actions, while CNV reflects temporal anticipation and prediction. Furthermore, it might be that as our participants become more certain about the best time to act, an RP component becomes weaker but a CNV component becomes stronger. Measured EEG would reflect the sum of these components, which cannot be separately identified. It is difficult to rule out this possibility. Although CNV and RP are traditionally studied using different experimental paradigms, they are not otherwise readily distinguishable (Brunia et al., 2011). Consistent with this, we compared scalp topographies for high certainty and low certainty actions in an exploratory analysis, and found no other notable differences beyond the greater peak amplitude around $\mathrm{FCz}$ for high certainty actions.

\section{Why is motor preparation so slow?}

Motor actions, even relatively complex reaching and grasping actions can be initiated within less than 200 milliseconds and completed accurately, and motor preparation takes only some of that time (Lara et al., 2018). The simple keypress required in RP studies should in principle require even less time to prepare. Neural computations are usually metabolically efficient (Hasenstaub et al., 2010). Why does the nervous system expand so much energy maintaining preparatory activity for so long? We have three hypotheses. First, although movement precision is not important in standard RP tasks, it is crucial in other contexts. By preparing movements well in advance whenever possible, the motor system may give itself time to correct for any inaccuracies before movement

510 begins (Churchland \& Shenoy, 2007). Second, slow motor preparation may leave time for upcoming actions to be modified or vetoed. This can happen if external factors mean that an action is no longer appropriate (Schultze-Kraft et al., 2016), or if internal feedback from predictive processing indicates that the action will have undesirable consequences. Third, while rapid changes in neural activity can occur, it may be that these abrupt changes are themselves metabolically costly. Thus, slow motor preparation might strike a balance between avoiding unnecessary prolonged periods of elevated firing rates and avoiding overly abrupt changes in firing rates. 


\section{Learning When to Act}

We presented participants with a novel temporal decision-making task. While there exists a large body of work on how humans and animals decide what action to produce (Bogacz, 2007; Edwards, 1954; O’Connell, Shadlen, Wong-Lin, \& Kelly, 2018), and how they learn about the value of alternative actions (Lee, Seo, \& Jung, 2012), less research focusses on deciding when to produce an action. Even then, work on decisions about when to act has almost exclusively focused on when agents cease sampling sensory evidence and commit to a decision (Cisek, Puskas, \& El-Murr, 2009; Drugowitsch, Moreno-Bote, Churchland, Shadlen, \& Pouget, 2012; Ratcliff, 1978). In contrast, our task is a pure timing task, in that one must decide when to perform an action, and there is no interaction between the when decision and the amount of evidence available to support the decision. In our task, the decision when to act is based on a model learned from previous experience, rather than on current sensory input. In fact, pure timing decisions of this kind are common in natural behaviour, and can be of vital importance. For instance, animals must decide how long to rest

530 between other activities such as foraging or hunting, and prey must decide how long to avoid an area after seeing a predator there. When two animals meet in confrontation, a stand-off often follows. Each animal must then compute when to attack, or run away etc. Human agents must decide not only when to remove food from the oven, but also when to change job, apply for promotion, start a family etc.

Traditionally, computational accounts of temporal decision-making have fallen into one of two categories. Some treat timing decisions as a series of discrete decisions about what to do, for instance deciding once per trial whether to continue pumping up a balloon, or not (Lejuez et al., 2002), Others treat temporal decision-making as simply a prior decision about how long to wait before acting, akin to time-based prospective memory (e.g. Misirlisoy \& Haggard, 2013). Here, we

540 treat it as a continuous-time decision about when to act. Doing so opens up a whole swathe of new questions. For instance, studies of continuous-space reinforcement learning - decisions about where to act - have shown that humans use a sophisticated and near-optimal approach to generalise across space (Wu, Schulz, Speekenbrink, Nelson, \& Meder, 2018). Moving through time is not like moving through space, since we move through time in only one direction, and at a constant rate. The computations underlying temporal decisions remain unclear, although models of neural timing are widespread (Paton \& Buonomano, 2018).

Finally, an important topic for future research will be to outline just how endogenous and exogenous causes of action interact in naturalistic decision-making. One promising approach is to consider how participants adjust the amount of external evidence they require before they commit to 
550 a decision (Bogacz et al., 2010; Cisek et al., 2009; Ratcliff, 1978). This is commonly captured by decision-models that include an endogenous urgency signal (Cisek et al., 2009) or by time-varying decision criteria (Bogacz et al., 2010). Urgency can increase as a function of elapsed time on a single trial. Self-initiated actions such as those required in our task might be caused by an urgency signal in the absence of any external evidence. Mechanisms such as these are likely to play an important role in any theory of endogenous and exogenous action.

\section{Conclusion}

Participants learned through trial and error when to make a simple action. As participants grew more certain about when to act, and became less variable and stochastic in the timing of their actions, the readiness potential prior to their actions became larger in amplitude. Our results are consistent with the proposal that the RP reflects motor planning or temporal expectation. They are harder to reconcile with the proposal that the RP is generated exclusively by random neural fluctuations in frontal cortical areas, though they are consistent with the presence of some stochastic contribution to the RP. These findings further speak against the theory that RP reflects uncertainty in the timing of action. This study raises new questions about the neural antecedents of self-initiated actions, and about the mechanisms underlying temporal decision-making. 


\section{References}

Alexander, P., Schlegel, A., Sinnott-Armstrong, W., Roskies, A. L., Wheatley, T., \& Tse, P. U. (2016). Readiness potentials driven by non-motoric processes. Consciousness and Cognition, 39(Supplement C), 38-47. https://doi.org/10.1016/j.concog.2015.11.011

BioSemi, B. (2011). BioSemi ActiveTwo.[EEG system]. Amsterdam: BioSemi.

Blakemore, Sarah-J., Wolpert, D. M., \& Frith, C. D. (1998). Central cancellation of self-produced tickle sensation. Nature Neuroscience, 1(7), 635-640. https://doi.org/10.1038/2870

Blakemore, Sarah-Jayne, Wolpert, D. M., \& Frith, C. D. (2002). Abnormalities in the awareness of action. Trends in Cognitive Sciences, 6(6), 237-242. https://doi.org/10.1016/S13646613(02)01907-1

Bogacz, R., Wagenmakers, E.-J., Forstmann, B. U., \& Nieuwenhuis, S. (2010). The neural basis of the speed-accuracy tradeoff. Trends in Neurosciences, 33(1), 10-16. https://doi.org/10.1016/j.tins.2009.09.002

Botvinick, M. M., Cohen, J. D., \& Carter, C. S. (2004). Conflict monitoring and anterior cingulate cortex: An update. Trends in Cognitive Sciences, 8(12), 539-546. https://doi.org/10.1016/j.tics.2004.10.003

Brembs, B. (2011). Towards a scientific concept of free will as a biological trait: Spontaneous actions and decision-making in invertebrates. Proceedings of the Royal Society B: Biological Sciences, 278(1707), 930-939. https://doi.org/10.1098/rspb.2010.2325

Brunia, C. H. M., Boxtel, G. J. M. van, \& Böcker, K. B. E. (2011). Negative Slow Waves as Indices of Anticipation: The Bereitschaftspotential, the Contingent Negative Variation, and the Stimulus-Preceding Negativity. The Oxford Handbook of Event-Related Potential Components. https://doi.org/10.1093/oxfordhb/9780195374148.013.0108

Churchland, M. M., \& Shenoy, K. V. (2007). Delay of movement caused by disruption of cortical preparatory activity. Journal of Neurophysiology, 97(1), 348-359. https://doi.org/10.1152/jn.00808.2006

Cisek, P., Puskas, G. A., \& El-Murr, S. (2009). Decisions in Changing Conditions: The UrgencyGating Model. Journal of Neuroscience, 29(37), 11560-11571. https://doi.org/10.1523/JNEUROSCI.1844-09.2009

Deecke, L., Scheid, P., \& Kornhuber, H. H. (1969). Distribution of readiness potential, pre-motion positivity, and motor potential of the human cerebral cortex preceding voluntary finger movements. Experimental Brain Research, 7(2), 158-168. https://doi.org/10.1007/BF00235441

Elsayed, G. F., Lara, A. H., Kaufman, M. T., Churchland, M. M., \& Cunningham, J. P. (2016). Reorganization between preparatory and movement population responses in motor cortex. Nature Communications, 7, 13239. https://doi.org/10.1038/ncomms13239

Erra, R. G., Arbotto, M., \& Schurger, A. (2019). An integration-to-bound model of decision-making that accounts for the spectral properties of neural data. Scientific Reports, 9(1), 1-12. https:// doi.org/10.1038/s41598-019-44197-0

Farrer, C., \& Frith, C. D. (2002). Experiencing Oneself vs Another Person as Being the Cause of an Action: The Neural Correlates of the Experience of Agency. NeuroImage, 15(3), 596-603. https://doi.org/10.1006/nimg.2001.1009

Friston, K. J., Daunizeau, J., Kilner, J., \& Kiebel, S. J. (2010). Action and behavior: A free-energy formulation. Biological Cybernetics, 102(3), 227-260. https://doi.org/10.1007/s00422-0100364-z

Frith, C. D., \& Haggard, P. (2018). Volition and the Brain - Revisiting a Classic Experimental Study. Trends in Neurosciences, 41(7), 405-407. https://doi.org/10.1016/j.tins.2018.04.009

Gershman, S. J. (2018). Deconstructing the human algorithms for exploration. Cognition, 173, 3442. https://doi.org/10.1016/j.cognition.2017.12.014 
Glimcher, P. W. (2005). Indeterminacy in brain and behavior. Annual Review of Psychology, 56, 25-56. https://doi.org/10.1146/annurev.psych.55.090902.141429

Gluth, S., Rieskamp, J., \& Büchel, C. (2013). Classic EEG motor potentials track the emergence of value-based decisions. NeuroImage, 79, 394-403. https://doi.org/10.1016/j.neuroimage.2013.05.005

Gramfort, A., Luessi, M., Larson, E., Engemann, D. A., Strohmeier, D., Brodbeck, C., Goj, R., Jas, M., Brooks, T., Parkkonen, L., \& Hämäläinen, M. (2013). MEG and EEG data analysis with MNE-Python. Frontiers in Neuroscience, 7. https://doi.org/10.3389/fnins.2013.00267

Grünewald, G., Grünewald-Zuberbier, E., Netz, J., Hömberg, V., \& Sander, G. (1979). Relationships between the late component of the contingent negative variation and the bereitschaftspotential. Electroencephalography and Clinical Neurophysiology, 46(5), 538545. https://doi.org/10.1016/0013-4694(79)90007-5

Haggard, P. (2017). Sense of agency in the human brain. Nature Reviews Neuroscience, 18(4), 196. https://doi.org/10.1038/nrn.2017.14

Hasenstaub, A., Otte, S., Callaway, E., \& Sejnowski, T. J. (2010). Metabolic cost as a unifying principle governing neuronal biophysics. Proceedings of the National Academy of Sciences, 107(27), 12329-12334. https://doi.org/10.1073/pnas.0914886107

Hughes, G., Schütz-Bosbach, S., \& Waszak, F. (2011). One Action System or Two? Evidence for Common Central Preparatory Mechanisms in Voluntary and Stimulus-Driven Actions. Journal of Neuroscience, 31(46), 16692-16699. https://doi.org/10.1523/JNEUROSCI.225611.2011

Hultin, L., Rossini, P., Romani, G. L., Högstedt, P., Tecchio, F., \& Pizzella, V. (1996). Neuromagnetic localization of the late component of the contingent negative variation. Electroencephalography and Clinical Neurophysiology, 98(6), 435-448. https://doi.org/10.1016/0013-4694(96)95507-8

Jahanshahi, M., Saleem, T., Ho, A. K., Dirnberger, G., \& Fuller, R. (2006). Random number generation as an index of controlled processing. Neuropsychology, 20(4), 391-399. https://doi.org/10.1037/0894-4105.20.4.391

Jenkins, I. H., Jahanshahi, M., Jueptner, M., Passingham, R. E., \& Brooks, D. J. (2000). Selfinitiated versus externally triggered movements. II. The effect of movement predictability on regional cerebral blood flow. Brain, 123(6), 1216-1228. https://doi.org/10.1093/brain/123.6.1216

Khalighinejad, N., Schurger, A., Desantis, A., Zmigrod, L., \& Haggard, P. (2018). Precursor processes of human self-initiated action. NeuroImage, 165(Supplement C), 35-47. https://doi.org/10.1016/j.neuroimage.2017.09.057

Lara, A. H., Elsayed, G. F., Zimnik, A. J., Cunningham, J. P., \& Churchland, M. M. (2018). Conservation of preparatory neural events in monkey motor cortex regardless of how movement is initiated. ELife, 7, e31826. https://doi.org/10.7554/eLife.31826

Lejuez, C. W., Read, J. P., Kahler, C. W., Richards, J. B., Ramsey, S. E., Stuart, G. L., Strong, D. R., \& Brown, R. A. (2002). Evaluation of a behavioral measure of risk taking: The Balloon Analogue Risk Task (BART). Journal of Experimental Psychology: Applied, 8(2), 75-84. https://doi.org/10.1037/1076-898X.8.2.75

Libet, B. (1985). Unconscious cerebral initiative and the role of conscious will in voluntary action. Behavioral and Brain Sciences, 8(4), 529-539. https://doi.org/10.1017/S0140525X00044903

Libet, B., Gleason, C. A., Wright, E. W., \& Pearl, D. K. (1983). Time of Conscious Intention to Act in relation to onset of Cerebral Activity (Readiness-Potential): The Unconscious Initiation of a Freely Voluntary Act. Brain, 106(3), 623-642. https://doi.org/10.1093/brain/106.3.623

Luce, R. D. (1959). Individual Choice Behavior: A Theoretical Analysis. Wiley. 
Maoz, U., Yaffe, G., Koch, C., \& Mudrik, L. (2019). Neural precursors of decisions that matterAn ERP study of deliberate and arbitrary choice. ELife, 8, e39787. https://doi.org/10.7554/eLife.39787

March, J. G. (1991). Exploration and Exploitation in Organizational Learning. Organization Science, 2(1), 71-87. https://doi.org/10.1287/orsc.2.1.71

Maye, A., Hsieh, C., Sugihara, G., \& Brembs, B. (2007). Order in Spontaneous Behavior. PLOS ONE, 2(5), e443. https://doi.org/10.1371/journal.pone.0000443

Murakami, M., Shteingart, H., Loewenstein, Y., \& Mainen, Z. F. (2017). Distinct Sources of Deterministic and Stochastic Components of Action Timing Decisions in Rodent Frontal Cortex. Neuron, 94(4), 908-919.e7. https://doi.org/10.1016/j.neuron.2017.04.040

Nachev, P., Kennard, C., \& Husain, M. (2008). Functional role of the supplementary and presupplementary motor areas. Nature Reviews Neuroscience, 9(11), 856-869. https://doi.org/10.1038/nrn2478

Nachev, P., Rees, G., Parton, A., Kennard, C., \& Husain, M. (2005). Volition and Conflict in Human Medial Frontal Cortex. Current Biology, 15(2), 122-128. https://doi.org/10.1016/j.cub.2005.01.006

Obhi, S. S., \& Haggard, P. (2004). Internally generated and externally triggered actions are physically distinct and independently controlled. Experimental Brain Research, 156(4), 518-523. https://doi.org/10.1007/s00221-004-1911-4

Parr, T., \& Friston, K. J. (2019). Generalised free energy and active inference. Biological Cybernetics, 113(5), 495-513. https://doi.org/10.1007/s00422-019-00805-w

Passingham, R. E. (1993). The frontal lobes and voluntary action. Oxford University Press.

Ratcliff, R. (1978). A theory of memory retrieval. Psychological Review, 85(2), 59.

Rohrbaugh, J. W., \& Gaillard, A. W. K. (1983). Sensory and Motor Aspects of the Contingent Negative Variation. In A. W. K. Gaillard \& W. Ritter (Eds.), Advances in Psychology (Vol. 10, pp. 269-310). North-Holland. https://doi.org/10.1016/S0166-4115(08)62044-0

Schultze-Kraft, M., Birman, D., Rusconi, M., Allefeld, C., Görgen, K., Dähne, S., Blankertz, B., \& Haynes, J.-D. (2016). The point of no return in vetoing self-initiated movements.

Proceedings of the National Academy of Sciences, 113(4), 1080-1085. https://doi.org/10.1073/pnas.1513569112

Schurger, A. (2018). Specific Relationship between the Shape of the Readiness Potential, Subjective Decision Time, and Waiting Time Predicted by an Accumulator Model with Temporally Autocorrelated Input Noise. Eneuro, ENEURO.0302-17.2018. https://doi.org/10.1523/ENEURO.0302-17.2018

Schurger, A., Sitt, J. D., \& Dehaene, S. (2012). An accumulator model for spontaneous neural activity prior to self-initiated movement. Proceedings of the National Academy of Sciences, 109(42), E2904-E2913. https://doi.org/10.1073/pnas.1210467109

Shibasaki, H., \& Hallett, M. (2006). What is the Bereitschaftspotential? Clinical Neurophysiology, 117(11), 2341-2356. https://doi.org/10.1016/j.clinph.2006.04.025

Tecce, J. J. (1972). Contingent negative variation (CNV) and psychological processes in man. Psychological Bulletin, 77(2), 73-108. https://doi.org/10.1037/h0032177

Thompson, W. R. (1933). On the Likelihood that One Unknown Probability Exceeds Another in View of the Evidence of Two Samples. Biometrika, 25(3/4), 285-294. JSTOR. https://doi.org/10.2307/2332286

Verleger, R., Haake, M., Baur, A., \& Śmigasiewicz, K. (2016). Time to Move Again: Does the Bereitschaftspotential Covary with Demands on Internal Timing? Frontiers in Human Neuroscience, 10. https://doi.org/10.3389/fnhum.2016.00642

Wallis, J. D. (2007). Orbitofrontal Cortex and Its Contribution to Decision-Making. Annual Review of Neuroscience, 30(1), 31-56. https://doi.org/10.1146/annurev.neuro.30.051606.094334 
Wise, S. P. (1985). The Primate Premotor Cortex: Past, Present, and Preparatory. Annual Review of Neuroscience, 8(1), 1-19. https://doi.org/10.1146/annurev.ne.08.030185.000245 